11. Huang S., Guo Y., Liu Y., Ke L., Liu G., Chen C. Study on the influence of water flow on temperature around freeze pipes and its distribution optimization during artificial ground freezing // Applied Thermal Engineering. - 2018. - V. 135, May. - P. 435-445. DOI: 10.1016/j.applthermaleng.2018.02.090.

12. Hu R., Liu Q., Xing Y. Case study of heat transfer during artificial ground freezing with groundwater flow // Water (Switzerland). - 2018. - V. 10, № 10. - Номер статьи 1322. DOI: 10.3390/w10101322.

13. Левин Л.Ю., Семин М.А., Зайцев А.В. Калибровка теплофизических свойств породного массива при моделировании формирования ледопородного ограждения строящихся шахтных стволов // Физикотехнические проблемы разработки полезных ископаемых. - 2019. - № 1. - С. 172-184.

14. Левин Л.Ю., Зайцев А.В., Семин М.А. Контроль теплового режима породного массива на основе применения оптоволоконных технологий мониторинга температур в скважинах // Горное эхо. - 2016. - № 1 (62). - C. 35-37.

УДК 622.253

DOI: 10.7242/ECHO.2019.1.18

\title{
УСТОЙЧИВОСТЬ КОНВЕКТИВНОГО ДВИЖЕНИЯ ВОЗДУХА В ШАХТНЫХ СТВОЛАХ
}

\author{
А.В. ШАЛИМОВ \\ Горный институт УрО РАН, г. Пермь
}

\begin{abstract}
Аннотация: В статье представлены результаты исследований устойчивости конвективного движения воздуха в шахтных стволах после отключения источника тяги. На основе численного моделирования установлено, что проветривание рудника естественной тягой нарушается путём образования протяжённых по глубине ствола воздушных вихрей. В приближении плоскопараллельного ламинарного течения несжимаемой среды с вертикальным градиентом температуры в поле силы тяжести определены поперечные профили скорости движения и температуры воздуха. Проведены аналитические вычисления устойчивости найденного течения к плоским длинноволновым возмущениям, в результате которых определено значение критического параметра Рэлея. Смоделирована поправка к коэффициенту объёмного расширения воздуха, позволяющая учесть гидростатическую сжимаемость воздуха. Получена зависимость для расчёта критического значения вертикального градиента температуры воздуха в стволе, превышение которого ведёт к образованию конвективных вихрей и нарушению сквозного проветривания.
\end{abstract}

Ключевые слова: рудник, ствол, естественная тяга, конвекция, депрессия, теплообмен, устойчивость, объемное расширение, гидростатическое сжатие.

Направление и интенсивность движения воздуха по горным выработкам определяется по совокупному действию всех источников тяги, к числу которых относится также и естественная тяга. В штатных режимах проветривания действие её не велико, составляет порядка $1 \%$ общей депрессии для рудников с одноуровневыми стволами и фигурирует в качестве поправок в вентиляционных расчётах. В случае возникновения пожара одной из возможных мер, принимаемых для ликвидации возгорания и недопущения распространения по выработкам продуктов горения, является останов вентилятора, после чего естественная тяга становится единственным источником поступления воздуха в рудник [1].

Осуществление прогноза проветривания рудника в подобной ситуации с помощью стандартных методов проведения вентиляционных расчётов невозможно по причине неоднозначности решений, реализуемость каждого из которых определяется совокупностью всех условий. Например, летом, когда наружный воздух более тёплый и менее плотный, чем рудничный, можно с уверенностью сказать, что естественной тяги не будет. Зимой же есть три сценария движения воздуха - прямой, нулевой и обратный, а интенсивность проветривания также под вопросом, т.к. зависит от величины общей тепловой депрессии, которая, в свою очередь, зависит от скорости движения воздуха через теплообмен. Неопределённость усугубляется наличием нескольких вентиляционных горизонтов, инициирующих появление тепловой межгоризонтной 
рециркуляции, зафиксированной на руднике РУ-3 ОАО «Беларуськалий» во время отключения ГВУ [2]. В результате разных значений тепловых депрессий в верхней и нижней части стволов проветривание верхнего горизонта не только прекращается, но и происходит опрокидывание воздушной струи.

Проблема неопределённости требует решения, т.к. необходимостью составления планов ликвидации аварий востребован хотя бы приблизительный прогноз движения воздушных потоков после отключения источника тяги. Модельный эксперимент в программной среде «SolidWorks» [3] показал, что по всей глубине ствола образуются протяжённые конвективные вихри, способствующие быстрому нагреву воздуха за счёт перемешивания и, как результат, уменьшению общей тепловой депрессии рудника и интенсивности его проветривания. При уменьшении аэродинамического сопротивления рудника, а также при увеличении температуры наружного воздуха, вихревая структура теряет силу и разрушается, давая путь сквозному проветриванию. Такой сценарий движения воздуха означает, что проблема неопределённости может быть разрешена только путём решения задачи устойчивости конвективного движения воздуха с получением оценочного критерия устойчивости.

Моделирование проводилось на основе теории устойчивости ламинарных конвективных течений лабораторных масштабов [4]. С целью получения аналитического решения исследовался профиль скорости движения воздуха с заданным расходом и продольным градиентом температуры в вертикальном плоском слое (рис. 1). В приближении модели турбулентности нулевого порядка с введением коэффициентов турбулентной вязкости и температуропроводности профиль стационарного течения воздуха выражается через турбулентное число Рэлея $R a$. Чем выше температурный градиент, тем больше $R a$, и тем больше кривизна профиля, вызванная конвективной подъёмной силой, при которой пристеночные нагретые слои воздуха поднимаются вверх, а менее нагретые по центру - опускаются вниз. Очевидно, что с увеличением горизонтальной стратификации скорости возрастает сдвиговое трение, и, следовательно, снижается устойчивость. А значит, при некотором критическом значении числа Рэлея профиль должен «сломаться», образовав конвективный вихрь.

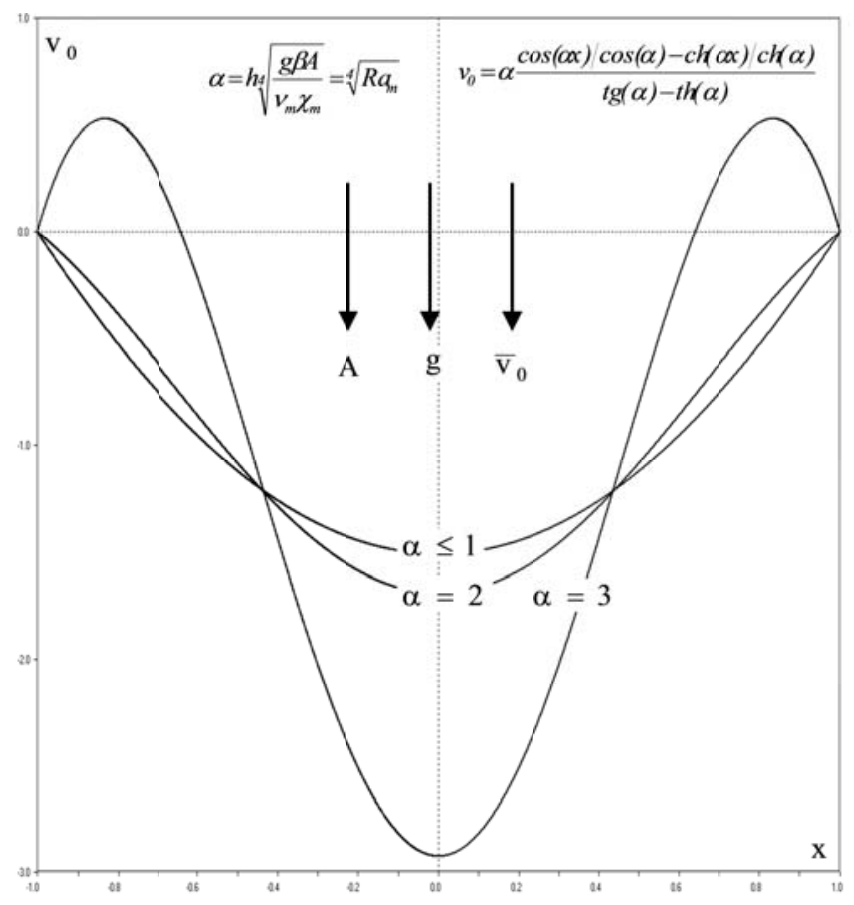

Рис. 1. Профили движения скорости воздуха в плоском вертикальном слое под действием вертикального градиента температуры 
Первой попыткой определения этого критического параметра было простое перенесение результатов решения задачи устойчивости конвективного ламинарного течения жидкости в лабораторных масштабах на крупномасштабное движения воздуха в стволах [1]. Задача устойчивости представленных профилей скорости движения воздуха решается следующими этапами:

1) Записывается система уравнений движения воздуха в безразмерных переменных: $[x, z]=h, \mathrm{м} ;[t]=h / \bar{v}_{0}, \mathrm{c} ;[v]=\bar{v}_{0}, \mathrm{M} / \mathrm{c} ;[T]=A h,{ }^{\circ} \mathrm{C} ;[p]=\rho_{0} \bar{v}_{0}^{2}, \Pi а$

$$
\left\{\begin{array}{c|c}
\frac{\partial \vec{v}}{\partial t}+(\vec{v} \nabla) \vec{v}=-\nabla p+\frac{\Delta \vec{v}}{R e_{m}}-\frac{G r_{m}}{\operatorname{Re}_{m}^{2}}\left(T-T_{\text {out }}\right) \frac{\vec{g}}{g} & \operatorname{Pr}_{m}=\frac{v_{m}}{\chi_{m}} \\
\frac{\partial T}{\partial t}+\vec{v} \nabla T=\frac{\Delta T}{\operatorname{Pr}_{m} \operatorname{Re}_{m}} & \operatorname{Re}_{m}=\frac{\bar{v}_{0} h}{v_{m}} \\
\operatorname{div}(\vec{v})=0 & G r_{m}=\frac{g \beta A h^{4}}{v_{m}^{2}}
\end{array}\right.
$$

$P r_{m}, R e_{m}, G r_{m}$ - турбулентные числа Прандтля, Рейнольдса и Грасгофа, безр.; $x$ и $z-$ горизонтальная и вертикальная координаты, безр.; $t$ - время, безр.; $h$ - полуширина слоя, м; $\bar{v}_{0}$ - средняя скорость движения воздуха, м/с; $T$ - температура воздуха, безр.; $T_{\text {out }}$ - температура наружного воздуха, безр.; $p$ - давление, безр.; $A$ - вертикальный градиент температуры, ${ }^{\circ} \mathrm{C} / \mathrm{M} ; \rho_{0}-$ средняя плотность воздуха, кг $/ \mathrm{M}^{3} ; g-$ ускорение свободного падения, м/ $\mathrm{c}^{2} ; v-$ скорость движения воздуха, безр.; $\beta-$ коэффициент объёмного расширения воздуха, $1 /{ }^{\circ} \mathrm{C} ; v_{m}$ и $\chi_{m}-$ коэффициенты турбулентной вязкости и температуропроводности, $\mathrm{M}^{2} / \mathrm{c}$.

2) На основное движение накладываются малые возмущения скорости, температуры и давления: $\vec{v}=\vec{v}_{0}+\vec{v}^{\prime}, \quad T=T_{0}+T^{\prime}, \quad p=p_{0}+p^{\prime}$, после чего система уравнений линеаризуется по малым возмущениям. Вводится функция тока $\psi$ для уменьшения количества уравнений:

$$
\left\{\begin{array}{c|c}
\frac{\partial}{\partial t} \Delta \psi+\left[v_{0} \frac{\partial}{\partial z} \Delta \psi-\frac{d^{2} v_{0}}{d x^{2}} \frac{\partial \psi}{\partial z}\right]=\frac{1}{\operatorname{Re}_{m}} \Delta \Delta \psi+\frac{G r_{m}}{\operatorname{Re}_{m}^{2}} \frac{\partial T^{\prime}}{\partial x} & v_{x}=\frac{\partial \psi}{\partial z} \\
\frac{\partial T^{\prime}}{\partial t}+v_{0} \frac{\partial T^{\prime}}{\partial z}+\frac{\partial T_{0}}{\partial x} \frac{\partial \psi}{\partial z}-\frac{\partial \psi}{\partial x}=\frac{\Delta T^{\prime}}{\operatorname{Pr}_{m} \operatorname{Re}_{m}} & v_{z}=-\frac{\partial \psi}{\partial x}
\end{array} .\right.
$$

3) Далее рассматриваются плоские нормальные возмущения $\psi(x, z)=\varphi(x) e^{-\lambda t+i k z}$, $T^{\prime}(x, z)=\theta(x) e^{-\lambda t+i k z}$, тип которых соответствует природе предполагаемой длинноволновой неустойчивости:

$$
\left\{\begin{array}{c}
-\lambda\left(\varphi^{\prime \prime}-k^{2} \varphi\right)+v_{0} i k\left(\varphi^{\prime \prime}-k^{2} \varphi\right)-v_{0}^{\prime \prime} i k \varphi=\frac{1}{\operatorname{Re}_{m}}\left(\varphi^{I V}-2 k^{2} \varphi^{\prime \prime}+k^{4} \varphi\right)+\frac{G r_{m}}{\operatorname{Re}_{m}^{2}} \theta^{\prime} \\
-\lambda \theta+v_{0} i k \theta+\tau_{0}^{\prime} i k \varphi-\varphi^{\prime}=\frac{1}{\operatorname{Pr}_{m} \operatorname{Re}_{m}}\left(\theta^{\prime \prime}-k^{2} \theta\right)
\end{array} .\right.
$$

4) Ищется граница неустойчивости для длинноволновой монотонной моды $(k=0$ и $\lambda=0): \varphi^{V}-R a_{m} \varphi^{\prime}=0$, где

$$
R a_{m}=G r_{m} P r_{m}=\frac{g \beta A h^{4}}{v_{m} \chi_{m}}
$$

5) Решение задачи на собственные значения даёт величину критического числа Рэлея

$$
R a_{m}^{(\kappa p)}=\pi^{4} \approx 100
$$

превышение которого в стволе означает начало развития внутристволовой конвекции. 
Но, как оказалось, при подстановке характерных значений всех физических параметров получается, что потеря устойчивости происходит при перепаде температуры воздуха 1 градус на километр по глубине, что реальности не соответствует, т.к. фактически означает, что устойчивости нет вообще, и малейший нагрев нарушает проветривание. Причина получения подобного не адекватного результата заключается в некорректности приближения не сжимаемости воздуха для объектов больших размеров. В отличие от лабораторных масштабов гравитация оказывает значительное влияние на исследуемый конвективный процесс по следующим двум причинам: 1) воздух сжимается под действием собственного веса, что увеличивает его плотность с глубиной, и оказывает стабилизирующее действие на устойчивость к восходящим конвективным потокам; 2) воздух разогревается в результате этого сжатия. С учётом указанных механизмов и теплообмена с крепью в программном комплексе «АэроСеть» в рамках одномерной модели были смоделированы изменения плотности воздуха с начальной температурой $+2{ }^{\circ} \mathrm{C}$ по глубине ствола при различных скоростях его движения [5]. Получается, что при скоростях движения воздуха более 10 м/мин тепловое расширение воздуха в результате теплообмена оказывается меньше его гидростатического сжатия, и плотность воздуха с глубиной растёт, а, значит, такое проветривание является устойчивым. Что касается меньших скоростей, то отрицательная вертикальная стратификация плотности возникает, например, для скорости 3 м/мин до глубины 400 метров, что означает возможность неустойчивости в этом случае. С глубиной интенсивность теплообмена уменьшается, и расширение сменяется сжатием (рис. 2).
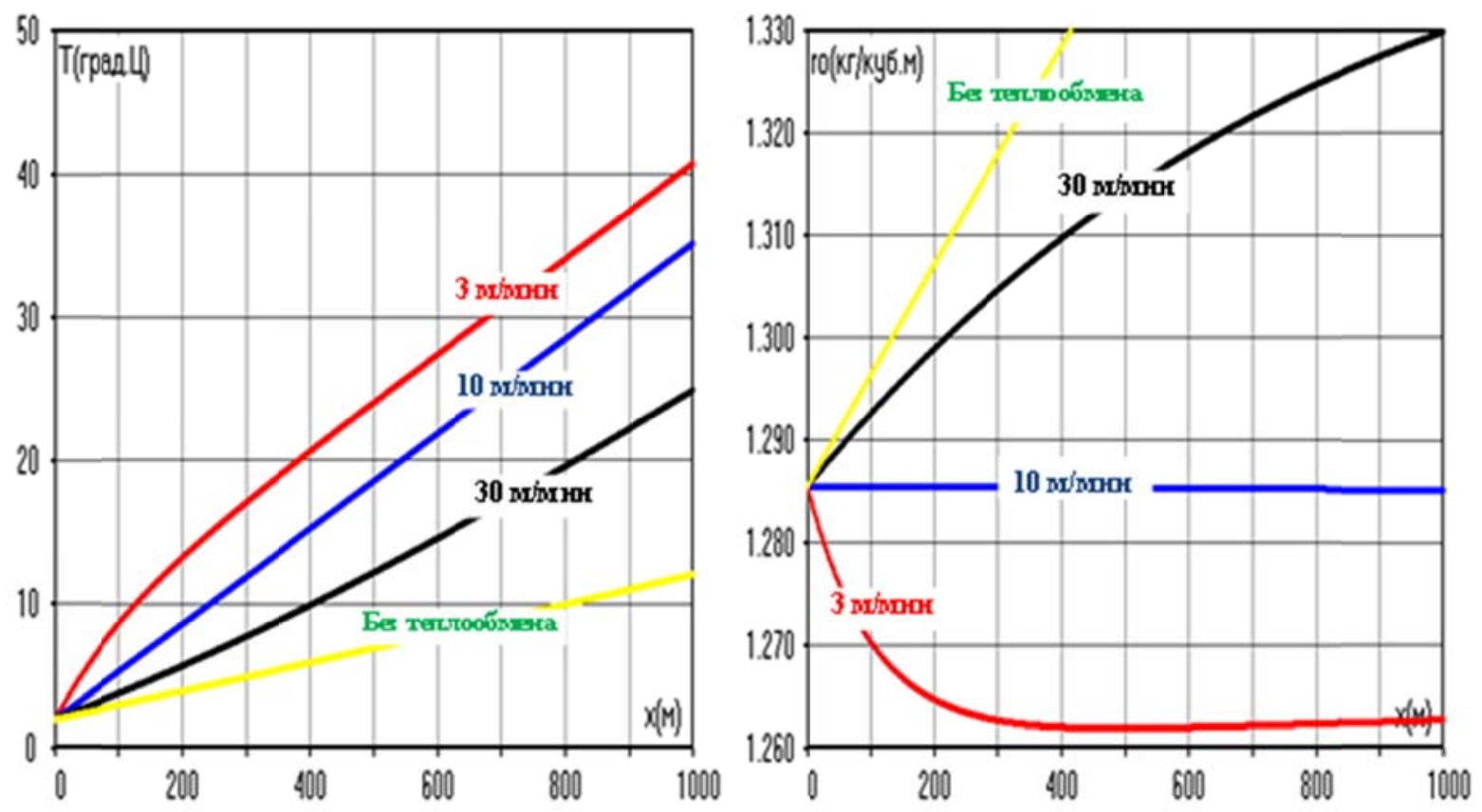

Рис. 2. Расчётные значения температуры и плотности воздуха по глубине воздухоподающего ствола в зависимости от скорости движения воздуха (начальная температура воздуха $+2^{\circ} \mathrm{C}$, среднегодовая температура местности +10 ${ }^{\circ} \mathrm{C}$, геотермическая ступень 30 м)

Учесть сжимаемость воздуха можно введением отрицательной стабилизирующей поправки к коэффициенту объёмного расширения в (1) 


$$
\beta \rightarrow \beta-\frac{g \mu}{(\gamma-1) R A \bar{T}}
$$

$\mu=0.029$ - молярная масса воздуха, кг/моль, $\gamma=1.4$ - показатель адиабаты, $R=8.31$ универсальная газовая постоянная, Дж/(моль $\cdot \mathrm{K}), \bar{T}=300$ - средняя температура воздуха, ${ }^{\circ}$ К. При этом способ определения критического значения числа Рэлея остаётся прежним (1), а результирующая зависимость с учётом (2) и (3) принимает вид, из которого вычисляется критический температурный градиент, инициирующий конвективную неустойчивость:

$$
A_{\kappa p}=\frac{\pi^{4} v_{m} \chi_{m}}{g \beta h^{4}}+\frac{g \mu}{(\gamma-1) R \beta \bar{T}} .
$$

При подстановке в (4) характерных значений параметров задачи скорректированное значение $A_{\kappa p}$ составляет примерно 8 градусов на 100 метров. Учитывая, что геотермический градиент для разных местностей находится в пределах от 1 до 10 градусов на 100 метров, можно заключить, что неустойчивость может возникать лишь в том случае, когда температура наружного воздуха значительно ниже среднегодовой температуры местности, определяющей температуру приповерхностного слоя породного массива.

Исходя из результатов проведённого оценочного расчёта, методика прогнозирования интенсивности проветривания рудника естественной тягой в аварийном режиме после отключения вентилятора представляется следующим образом. В программном комплексе «АэроСеть» производится расчёт температуры и скоростей установившегося движения. Если значение вертикального температурного градиента воздуха в верхней части ствола не превышает критического значения, то результат прогноза считается достоверным. В противном случае прогноз не верен и требует корректировки в области неустойчивости методами трёхмерного моделирования.

Работа выполнена при поддержке гранта по конкурсу фундаментальных исследований Уральского Отделения Российской Академии Наук (проект № 18-5-5-5).

\section{БИБЛИОГРАФИЧЕСКИЙ СПИСОК}

1. Kazakov B.P., Shalimov A.V., Semin M.A. Stability of natural ventilation mode after main fan stoppage // International Journal Heat Mass Transfer. - 2015. - V. 86. - P. 288-293. DOI: 10.1016/j.ijheatmasstransfer.2015.03.004.

2. Казаков Б.П., Шалимов А.В. О возможности проветривания рудника естественной тягой после отключения главной вентиляционной установки // Изв. вузов. Горн. журн. - 2013. - № 2. - С. 59-65.

3. Казаков Б.П., Левин Л.Ю., Шалимов А.В., Зайцев А.В. Разработка энергосберегающих технологий обеспечения комфортных микроклиматических условий при ведении горных работ // Записки Горного института. - 2017. - Т. 223. - С. 116-124.

4. Гершуни Г.3., Жуховицкий Е.М., Непомнящий А.А. Устойчивость конвективных течений. - М.: Наука, 1989. - 318 с.: ил.

5. Казаков Б.П., Шалимов А.В., Сёмин М.А., Гришин Е.Л., Трушкова Н.А. Конвективная стратификация воздушных потоков по сечению горных выработок, ее роль в формировании пожарных тепловых депрессий и влияние на устойчивость проветривания // Горный журнал. - 2014. - №12. - С. 105-109. 